\title{
Acute focal Myocarditis with Preserved Ventricular Function Masquerading as Acute ST-elevation Inferior Wall Myocardial Infarction
}

\begin{abstract}
Myocarditis is a pathological inflammatory condition which can result in significant damage to the myocardium and more specifically the myocytes. Acute myocarditis can present similarly to myocardial infarction, and can rise to the top of the differential diagnosis when chest pain, cardiac enzymes, and ECG that suggest myocardial infarction are followed by a normal coronary angiogram. The gold standard of diagnosis for myocarditis is an endomyocardial biopsy as a positive biopsy is very specific; however, the inflammation of myocarditis may be diffuse or focal, limiting the biopsy's sensitivity. We present a case of a 19 year old man with acute focal myocarditis masquerading as an acute ST-elevation inferior wall myocardial infarction on ECG with elevated troponin I. This is an unusual presentation as the patient's echocardiogram and cardiac catheterization did not show ventricular dysfunction. Our diagnosis was confirmed by cardiac MRI rather than endomyocardial biopsy, due to the high risk of adverse events associated with the latter.
\end{abstract}

Acute myocarditis that mimicks a myocardial infarction in presentation is a rare, but well known occurrence [1]. Acute myocarditis typically presents with a less distinct prodromal illness period, in contrast with fulminant myocarditis, which has a more defined viral prodrome and associated with more severe cardiovascular compromise. Acute myocarditis is also more likely to present in those patients with established ventricular dysfunction, with the potential to progress to dilated cardiomyopathy [2].

Acute focal myocarditis without ventricular dysfunction is an uncommon presentation that has become more recognized recently as new imaging modalities allow this rare presentation to be differentiated.

Keywords: Myocarditis; ST elevation MI; STEMI

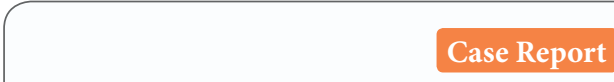

Volume 2 Issue 3 - 2015

Matthew Cholankeril, Jeffy Kalavelil, Vikram Doraiswamy, Joaquim Correia and Abbas Shehadeh*

Department of Cardiology, Seton Hall University School of Graduate Medical Education, USA

*Corresponding author: Abbas Shehadeh, Saint Michael ‘s Medical Center, Department of cardiology and Cardiovascular research, Newark, New Jersey, USA, Email: abbasshehadeh@comcast.net

Received: February 05, 2015 | Published: April 01, 2015

\section{Case Report}

A 19 year old male with no significant past medical history, presents to the emergency room with sudden onset, severe, retrosternal, chest pain while in school. The patient stated the pain was constant, $6 / 10$ severity, lasting 6 hours prior to presentation, no radiation or alleviating or aggravating factors, and associated with initial shortness of breath at rest and diaphoresis. The patient has no history of any prior episodes of similar presentation. The patient has no known drug allergies, and was not taking any medications. The patient's family history is significant for his mother having a history of Sjogren's and surviving an acute myocardial infarction with cardiac arrest at age 42 , who received a drug-eluting stent after the MI. The patient denies any smoking, alcohol, drug use and reports a noncontributory sexual history.

On admission, the patient was afebrile, with a blood pressure of $118 / 72$, pulse rate of 78 beats per minute, respiratory rate of 16 breaths per minute, and oxygen saturation of $98 \%$ on room air. The physical exam indicated his jugular venous pressure was within normal limits. His heart rate and rhythm were regular, with no murmurs, rubs, or gallops appreciated, and normal S1 and S2 heart sounds. In addition, his lungs were clear to auscultation bilaterally, and the exam of his extremities revealed no appreciable peripheral edema. Chest X-ray revealed a normalsized cardiac border with no infiltrates or effusions.

A diagnosis of acute inferior wall ST-elevation myocardial infarction was initially considered due to the ECG findings, which showed upwardly concave ST elevations in leads II, III, and aVF, subtle PR depressions in leads II, III, and aVF, non specific ST elevations in V3-V5, T wave inversions in $\mathrm{VVR}, \mathrm{aVL}, \mathrm{V} 1$, and V2, as well as reciprocal ST depression in aVR and V (Figure 1a).

At this time, his initial cardiac enzymes revealed peak values of a troponin I $4.56 \mathrm{ng} / \mathrm{ml}$ (upper limit of normal $=0.06 \mathrm{ng}$ / $\mathrm{ml}$ ), myocardial band fraction was $36 \mathrm{ng} / \mathrm{ml}$ (upper limit of normal=6.3 ng/ml), and creatinine kinase $494 \mathrm{IU} / \mathrm{L}$ (upper limit of normal=195 IU/L), with a normal basic metabolic profile and complete blood count. After the patient was given nitroglycerin without resolution of his chest pain, he was started on IV heparin, aspirin, clopidogrel, statin, beta-blockers, ace-inhibitors, and low dose intravenous morphine in the emergency room, and reported improvement of the chest pain. Echocardiography performed during the initial admission revealed an ejection fraction of $60 \%$ with no regional wall motion abnormalities, no valvular dysfunction, no pericardial effusion, and no diastolic dysfunction. Due to the patient's history of present illness, family 
history, and ECG findings, he was emergently taken for cardiac catheterization, which revealed normal coronary angiography with no abnormalities.

On the second day of his hospitalization, the patient's cardiac enzymes trended down with a troponin I $1.34 \mathrm{ng} / \mathrm{ml}$ (upper limit of normal $=0.06 \mathrm{ng} / \mathrm{ml}$ ), myocardial band fraction was 9.6 $\mathrm{ng} / \mathrm{ml}$ (upper limit of normal=6.3 ng $/ \mathrm{ml}$ ), and creatinine kinase $123 \mathrm{IU} / \mathrm{L}($ upper limit of normal=195). A calcium pyrophosphate imaging exam was done and was negative for myocardial scarring. A repeat ECG showed improvement of the initial ST segment and T wave changes, with T wave inversions in leads II, III, aVF (Figure 1b).

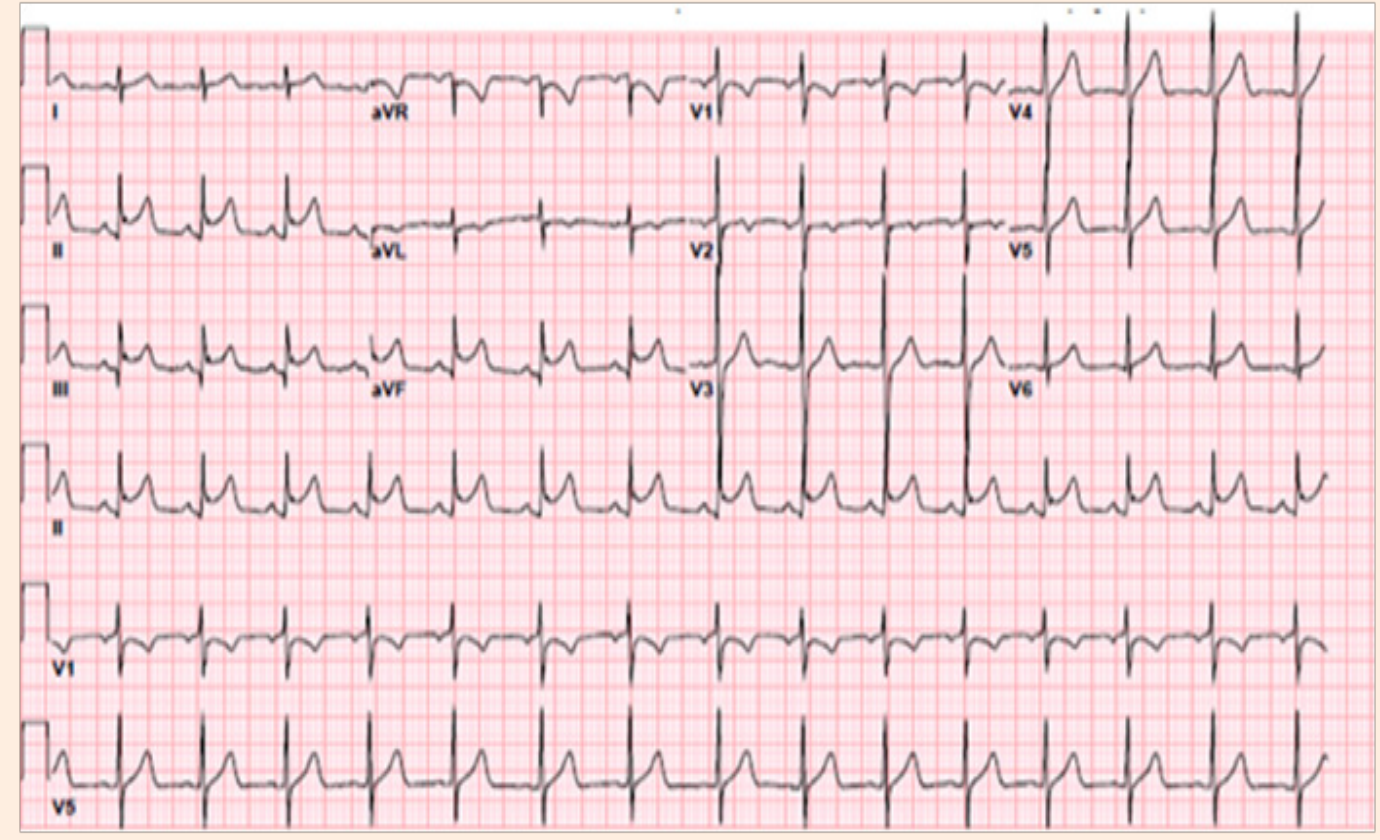

Figure 1a: ECG findings, which showed upwardly concave ST elevations in leads II, III, and aVF, subtle PR depressions in leads II, III, and aVF, non specific ST elevations in V3-V5, T wave inversions in aVR, aVL, V1, and V2, as well as reciprocal ST depression in aVR and V1.

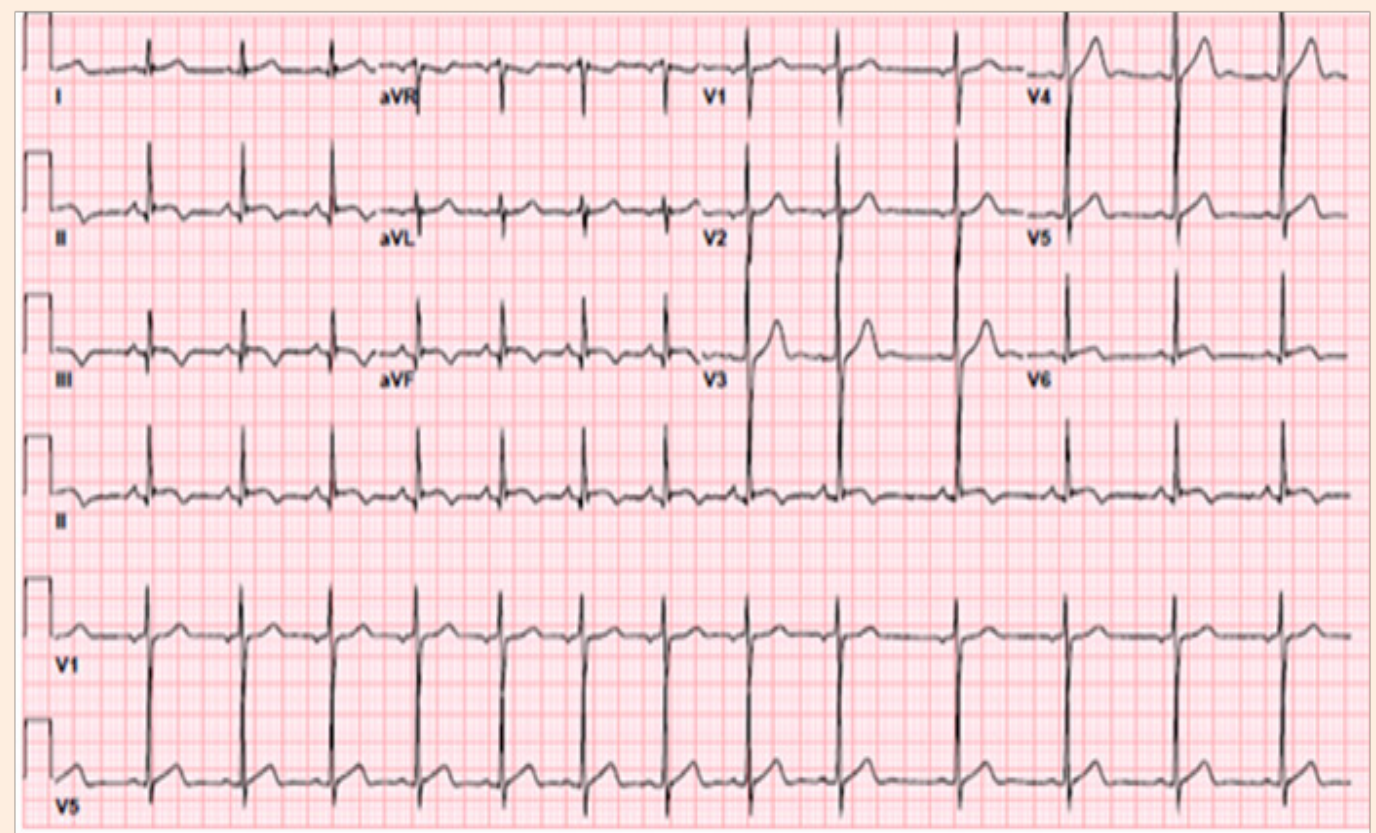

Figure 1b: Repeat ECG showed improvement of the initial ST segment and T wave changes, with T wave inversions in leads II, III, aVF.

Citation: Cholankeril M, Kalavelil J, Doraiswamy V, Correia J, Shehadeh A (2015) Acute focal Myocarditis with Preserved Ventricular Function Masquerading as Acute ST-elevation Inferior Wall Myocardial Infarction. J Cardiol Curr Res 2(3): 00064. DOI: 10.15406/jccr.2015.02.00064 
The patient was then taken for a cardiac magnetic resonance imaging exam which revealed an ejection fraction of $60 \%$, delayed enhancement involving the mid lateral wall, a portion of the inferior wall, and a portion of the epicardial wall in the inferolateral area, consistent with focal myocarditis in the inferolateral wall (Figure 2a), (white arrow).

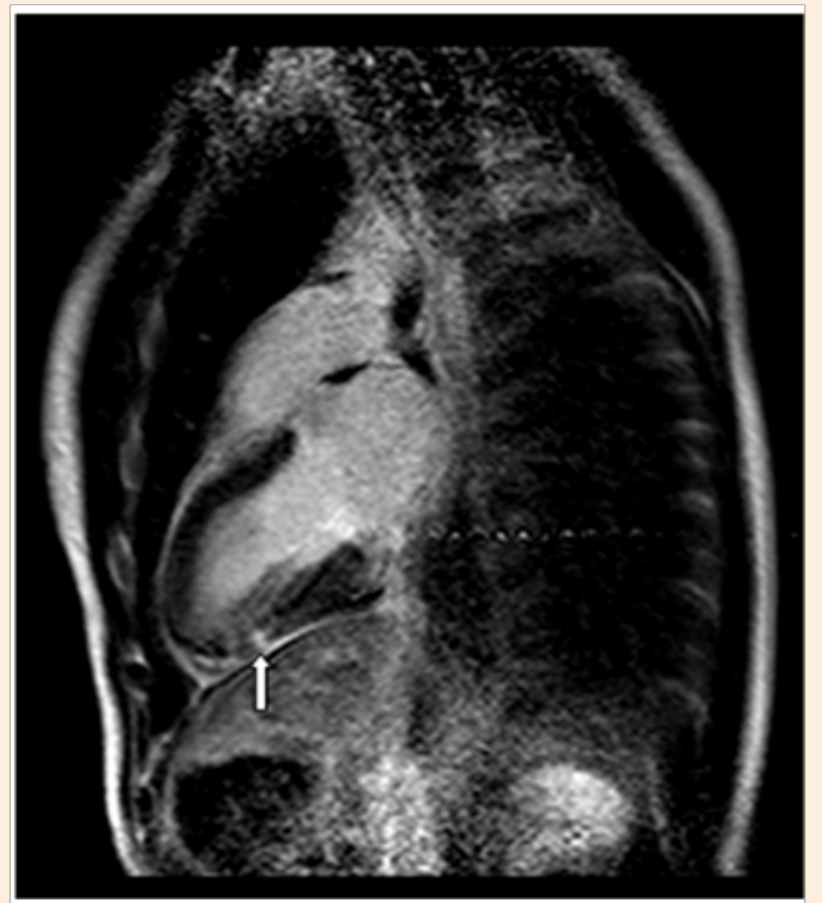

Figure 2a: Cardiac magnetic resonance imaging exam which revealed an ejection fraction of $60 \%$, delayed enhancement involving the mid lateral wall, a portion of the inferior wall, and a portion of the epicardial wall in the inferolateral area, consistent with focal myocarditis in the inferolateral wall(white arrow).

Testing was done for collagen vascular diseases, as well as a Mantoux test, all of which were negative. The patient's ESR was also within the normal range, consistent with the focal nature of his inflammatory process that did not mount a systemic response. On the third day of hospital admission, the patient's symptoms had resolved, with normalization of his cardiac enzymes. He was discharged without any new medications, and followed up with a repeat cardiac MRI in two months that showed no abnormalities (Figure $2 \mathrm{~b}$ ). The patient reported no recurrence of his chest pain.

\section{Discussion}

Acute myocarditis can masquerade as a myocardial infarction when one is evaluating the patient's history, physical, laboratory, and ECG findings. Once a cardiac catheterization has ruled out coronary disease, myocarditis should be considered a likely diagnosis. This diagnosis can be confirmed with endomyocardial biopsy; however, it is a relatively high risk invasive procedure, with limited contribution to the differential diagnosis, particularly in focal myocarditis. The cardiac MRI for our patient showed myocarditis that involved the epicardium and spared the endomyocardium that is affected in a myocardial infarction
[3]. A study done by Gahide and colleagues found that delayed enhancement cardiac MRI, consistent with the imaging done in this case, was able to demonstrate epicardial involvement in acute myocarditis, and reverse previously incorrect diagnoses of myocarditis that were myocardial infarction [4].

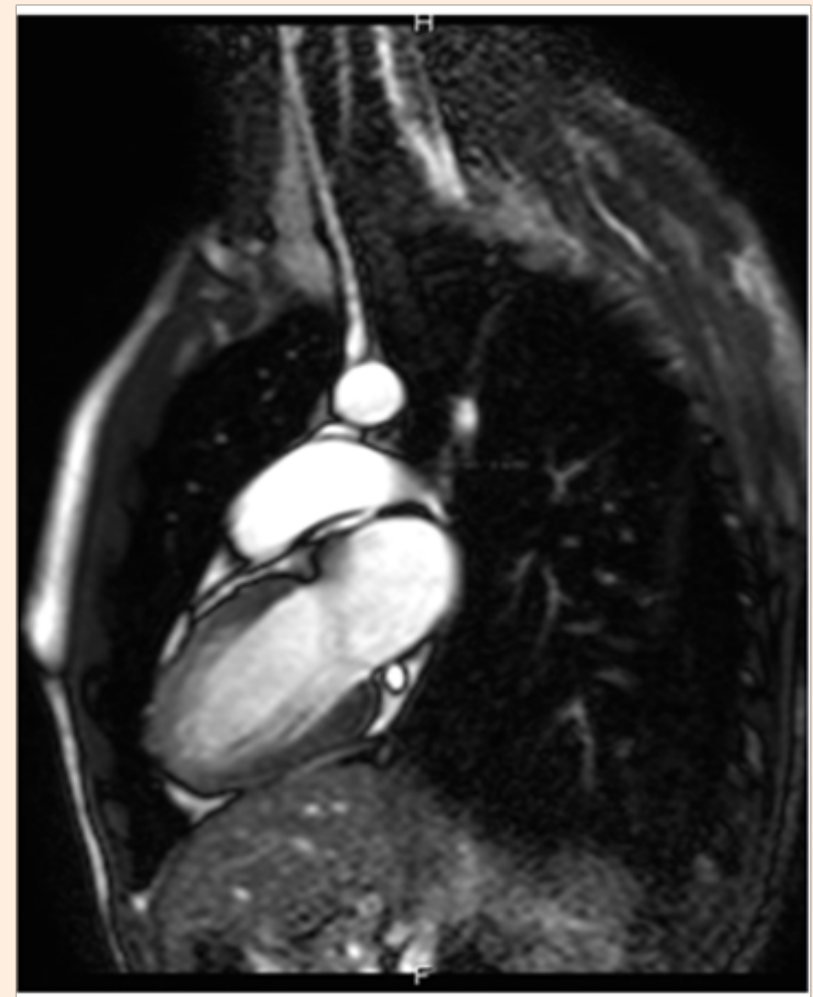

Figure 2b: Repeat cardiac MRI in two months that showed no abnormalities.

Typically, acute myocarditis presents with left ventricular dysfunction and can later progress to dilated cardiomyopathy. However, in this case left ventricular function was intact. Acute myocarditis with intact left ventricular function is an uncommon occurrence, but has been citied in earlier literature reports. In a study by Dec et al. [5] left ventricular function was normal in six of eleven patients diagnosed with myocarditis on biopsy. $5 \mathrm{New}$ imaging modalities hold promise in evaluating patients with acute myocarditis and preserved ejection fraction. In a study by Hsiao et al. [6] Strain (S) and Strain Rate (SR) imaging, measured by 2-D speckle tracking echocardiogram (2D-STE) were assessed in patients with imaging showing acute myocarditis and preserved left ventricular ejection fraction. The study showed significant differences between the two groups; septal thickness, LV end-systolic dimension (LVESD), and EF were significantly lower in the myocarditis group $(\mathrm{p}<0.05)$ using the 2D STE method. Thus, this method may help more thoroughly evaluate patients who initially present with acute myocarditis. Although our patient who presented had normal ventricular function, it may have improved by the time systolic function was evaluated in the cardiac catheterization laboratory and a more specific test such as Tissue Doppler with 2D STE could have been helpful. A cardiac catheterization can be helpful in patients with 
EKG changes of upwardly convex ST elevations and low ejection fraction on echocardiogram.

\section{Conclusion}

In summary, acute myocarditis can present similarly to acute myocardial infarction, and should be considered in the setting of a normal coronary angiogram. Although acute myocarditis typically presents with ventricular dysfunction, normal ejection fractions in the setting of acute myocarditis can occur, especially in acute focal myocarditis. However, this may reflect a normalization of the ventricular function by the time of evaluation, so a more specific test such as 2D-STE or more immediate cardiac catheterization can be useful.

\section{References}

1. Miklozek CL, Crumpacker CS, Royal HD, Come PC, Sullivan JL, et al. (1988) Myocarditis presenting as acute myocardial infarction. Am Heart J 115(4): 768-776
2. Lieberman EB, Hutchins GM, Herskowitz A, Rose NR, Baughman KL (1991) Clinicopathologic description of myocarditis. J Am Coll Cardiol 18(7): 1617-1626.

3. Testani JM, Kolansky DM, Litt H, Gerstenfeld EP (2006) Focal Myocarditis Mimicking Acute ST-Elevation Myocardial Infarction. Tex Heart Inst J 33(2): 256-259.

4. Gahide G, Bertrand D, Roubille F, Tron C, Skaik S, et al. (2010) MR delayed enhancement imaging findings in suspected acute myocarditis. Eur Radiol 20(1): 65-72.

5. Dec GW Jr, Waldman H, Southern J, Fallon JT, Hutter AM, et al. (1992) Viral myocarditis mimicking acute myocardial infarction. J Am Coll Cardiol 20(1): 85-89.

6. Hsiao J, Bonnichsen CR, Koshino Y, Saleh HK, Yu Y, et al. (2010) Left Ventricular Deformation by 2-D Speckle Tracking Echocardiogram for the Diagnosis of Acute Myocarditis With Preserved LV Ejection Fraction. Circulation 122: A20570. 